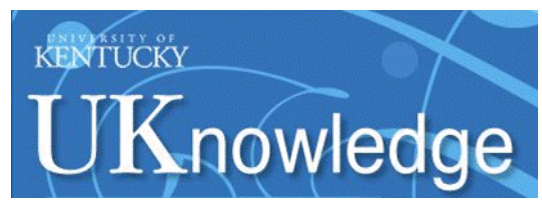

University of Kentucky

UKnowledge

$10-2017$

\title{
Secondhand Smoke Exposure and Preclinical Markers of Cardiovascular Risk in Toddlers
}

\author{
Judith A Groner \\ American Academy of Pediatrics \\ Hong Huang \\ University of Kentucky, hong.huang@uky.edu \\ Mandar S. Joshi \\ University of Kentucky \\ Nicholas Eastman \\ University of Kentucky \\ Lisa Nicholson \\ The Ohio State University
}

See next page for additional authors

Follow this and additional works at: https://uknowledge.uky.edu/pediatrics_facpub

Part of the Cardiology Commons, and the Pediatrics Commons

Right click to open a feedback form in a new tab to let us know how this document benefits you.

\section{Repository Citation}

Groner, Judith A; Huang, Hong; Joshi, Mandar S.; Eastman, Nicholas; Nicholson, Lisa; and Bauer, John Anthony, "Secondhand Smoke Exposure and Preclinical Markers of Cardiovascular Risk in Toddlers" (2017). Pediatrics Faculty Publications. 297.

https://uknowledge.uky.edu/pediatrics_facpub/297

This Article is brought to you for free and open access by the Pediatrics at UKnowledge. It has been accepted for inclusion in Pediatrics Faculty Publications by an authorized administrator of UKnowledge. For more information, please contact UKnowledge@lsv.uky.edu. 


\section{Secondhand Smoke Exposure and Preclinical Markers of Cardiovascular Risk in Toddlers}

Digital Object Identifier (DOI)

https://doi.org/10.1016/j.jpeds.2017.06.032

Notes/Citation Information

Published in The Journal of Pediatrics, v. 189, p. 155-161.

(C) 2017 Elsevier Inc. All rights reserved.

This manuscript version is made available under the CC-BY-NC-ND 4.0 license https://creativecommons.org/licenses/by-nc-nd/4.0/.

The document available for download is the author's post-peer-review final draft of the article.

Authors

Judith A Groner, Hong Huang, Mandar S. Joshi, Nicholas Eastman, Lisa Nicholson, and John Anthony Bauer 


\title{
Secondhand smoke exposure and preclinical markers of cardiovascular risk in toddlers
}

\author{
Judith A. Groner, MD ${ }^{1,2}$, Hong Huang, PhD $^{1,3}$, Mandar S. Joshi, $\mathrm{PhD}^{3}$, Nicholas Eastman, \\ BS $^{3}$, Lisa Nicholson, $\mathbf{P h D}^{2}$, and John Anthony Bauer, $\mathbf{P h D}^{1,3}$ \\ ${ }^{1}$ AAP Julius B. Richmond Center of Excellence, Elk Grove Village, IL, USA; \\ ${ }^{2}$ Nationwide Children's Hospital, Department of Pediatrics, The Ohio State University College of \\ Medicine, Columbus, $\mathrm{OH}$, USA, \\ ${ }^{3}$ Kentucky Children's Hospital, Department of Pediatrics, University of Kentucky College of \\ Medicine, Lexington, KY, USA.
}

\begin{abstract}
Objective: Links between secondhand smoke (SHS) exposure and cardiovascular disease in adults are well established but seldom reported during childhood. Although rates of smoking have decreased, young children from low-income backgrounds remain likely to be exposed to SHS. The purpose of this study was to investigate relationships between SHS exposure in young children and several preclinical markers of cardiovascular risk that have been established as relevant to adult populations.
\end{abstract}

Methods: 139 children, 2-5 years of age, were enrolled in a cross-sectional study. SHS exposure was objectively determined by hair nicotine level; a comprehensive panel of clinical markers (AM blood pressure, fasting glucose $\&$ insulin, lipid profiles, inflammation) and research markers (markers of oxidation, endothelial stress, and endothelial repair) of cardiovascular risk status were assessed. Univariate and multivariate linear regression were used to evaluate relationships between SHS exposure and cardiovascular risk markers.

Results: Hair nicotine levels were directly correlated with blood pressure and serum CRP, and inversely correlated with serum HDL and endothelial cell progenitor cell prevalence. In multivariate analyses, these relationships remained when controlled for age, sex, BMI z-score, maternal education, and method of payment. Additionally, in multivariate analyses, hair nicotine level was significantly negatively correlated with total anti-oxidant capacity.

Conclusions: These results support the view that SHS exposure in the very young has a detectable relationship with several markers of cardiovascular risk, long before the emergence of clinical disease. Further studies to define mechanisms and strategies to prevent and mitigate these risks early in life are warranted.

Address Correspondence to: Judith A Groner MD, Nationwide Children's Hospital, 700 Children's Drive, Columbus, Ohio, 43205. Phone: 614-722-4957, Fax: 614-722-4966, Judith.groner@ nationwidechildrens.org.

Conflict of Interest Statement: We have no conflict of interest to disclose. 


\section{BACKGROUND:}

The latest CDC report shows that despite an overall reduction in secondhand smoke (SHS) exposure from 1999 to $2015,25 \%$ of the US population remains exposed ${ }^{1}$. Of special concern are children from low-income homes and African-American children, since they have the highest rates of biologically measured SHS exposure ${ }^{1}$. Over $40 \%$ of US children aged 3-11 were exposed to tobacco smoke from 2011-2012, based on a biological marker of exposure, serum cotinine levels ${ }^{1}$. An inverse relationship between socioeconomic status and SHS exposure has been well documented ${ }^{2,3}$, and recent analyses have shown that for every decrease in family income ratio, serum cotinine levels increased by $1.18 \mathrm{ng} / \mathrm{L}$ among children ${ }^{2}$. This evidence, coupled with a burden of lifetime exposure make young children of utmost concern. Better defining health risks of SHS in young children from low-income settings may help to underscore this health-risk disparity and help pediatricians to enhance strategies to mitigate these risks.

Links between SHS and cardiovascular disease (CVD) and death in adults are very well established $^{4-7}$. SHS is a known risk factor for the development of atherosclerotic heart disease and increases the risk of CVD by about $30 \%$ in non-smoking adults ${ }^{5-7}$. In contrast to compelling evidence in adults studies demonstrating cardiovascular implications of SHS exposure during childhood are less defined. Young children are at particular risk for SHS exposure $^{2,8}$. More importantly, in our previous work, we demonstrated that toddlers had higher hair nicotine levels when compared to older children with equivalent survey measures of SHS exposure?

A challenge for studying cardiovascular risk in pre-adulthood is the fact that children and adolescents very rarely exhibit clinical manifestations of acquired (non-congenital) heart disease. Most adult forms of cardiovascular disease are either related to or driven by inflammatory processes ${ }^{4}$, and such processes are apparently initiated in childhood. However, few studies have assessed the concurrent relationship between SHS exposure and cardiovascular risk markers in children younger than six years old ${ }^{10}$.

Our goal was to better define the relationship between SHS exposure in toddlers age 2-5 and preclinical cardiovascular disease risk. We investigated several surrogate markers of CVD risks in relation to SHS exposure and included traditional clinical measures as well as research measures recognized as relevant and mechanistically involved in adult settings. Clinical measures were blood pressure, glucose metabolism, blood lipids, and markers of inflammation, while 'research measures' were markers oxidation, and vascular endothelial stress and endothelial repair. Each of these indicators has been linked to SHS exposure and cardiovascular disease in adult or older pediatric populations ${ }^{7,11-14}$ but has not been investigated in very young children. Prior studies have not focused on this age group where cardiovascular implications of SHS may be importantly under-recognized. 


\section{METHODS:}

\section{Human Subject Recruitment:}

The Nationwide Children's Hospital (NCH) IRB approved of the study. Participants were children aged 2-5 years and parents provided informed consent. They were recruited via convenience sampling through recruiting in Nationwide Children's Hospital Primary Care Network (Columbus, Ohio), and advertising via an internal hospital e-mail system. The Primary Care Network primarily serves low-income, urban children in Columbus. Inclusion criteria were healthy children both exposed and unexposed to SHS by parental report. Exclusion criteria were: the presence of one or more of the following: acute febrile illness or other active infections, congenital heart disease, diabetes (Type 1 or 2), elevated fasting glucose (>100 mg/dl), family history of elevated cholesterol, use of oral or inhaled (antiinflammatory) steroids within one month of testing, and/or not having enough hair for hair sampling of SHS exposure (hair nicotine level). This approach to enrollment thus avoided children with persistent asthma, because of the use of daily anti-inflammatories, which we considered confounding for this present study.

\section{Study Procedures:}

The study was introduced to the mother or caregiver at a clinic visit. Subjects were subsequently scheduled for testing at a research site in the morning between 8-10am, after overnight fasting. The protocol was carried out as follows: a) Study procedures were described with parental informed consent and youth/teen assent and consent obtained, b) anthropomorphic measurements obtained, c) structured interview with subject and parent (demographics and SHS exposure history), d) hair sample obtained and e) the blood sample of $7 \mathrm{ml}$ was collected for biomarkers and covariates. After serum sample collection all assays were stored on ice and used within $12 \mathrm{hrs}$ of collection ( $24 \mathrm{hr}$ for flow cytometry studies).

\section{Height, Weight, BMI:}

Height and weight were obtained using a Tanita BWB800 scale and Seca stadiometer. Heights were measured to the nearest $0.5 \mathrm{~cm}$, weights to the nearest $0.1 \mathrm{~kg}$. BMI's and zscore norms were determined according to CDC guidelines (http://www.cdc.gov/ healthyweight/assessing/bmi/childrens_bmi/about_childrens_bmi.htmlref).

\section{Measured variables:}

We assessed a comprehensive panel of clinically relevant and research-based outcome variables that have been used by others to reflect cardiovascular status and related disease risks. Brief descriptions of methods and rationale are shown below. Additional analytical details are provided in online supplemental materials.

\section{Secondhand smoke exposure:}

Hair nicotine was used as a biological marker of SHS exposure to assess long-term evaluation of smoke exposure since the nicotine is incorporated into the growing hair shaft over several months. Samples are easy to obtain, handle, and store. Approximately 20-40 
hair shafts from the occipital area were cut at the root and $2-3 \mathrm{~cm}$ in length. Hairs were stored and later sent for assay at an established contract research facility (Johns Hopkins School of Public Health). The hair nicotine assay involves washing the hair sample before analysis and therefore is designed to measure inhaled nicotine, and not ambient nicotine simply adhered to hair. Content is expressed as $\mathrm{ng} / \mathrm{mg}$ of hair.

\section{Blood pressure:}

Blood pressure was measured via a Critikon-Dinamap Compact $\mathrm{T}$ monitor. The subject was fasting and allowed upright (typically in a parent's lap) for at least 5 minutes, the measurement is taken on the subject's left arm while. Normalized BP (percentile of normative values) was derived from NHLBI tables, accounting for height, age, and gender (http://www.nhlbi.nih.gov/guidelines/hypertension/child_tbl.htm).

\section{Blood lipid profiles, glucose, insulin:}

Fasting lipid profiles and glucose were measured via our hospital core lab facility. Insulin was determined by EIA (GenWay Biotech Inc. San Diego, CA, Cat\# 40-056-205011).

\section{Biomarkers of inflammation:}

C-reactive protein (CRP): This was measured as a classical and clinically relevant measure of systemic inflammation that has been linked to SHS exposure in both adults and children ${ }^{11,15}$. Elevations in serum CRP, and increased gene expression in the myocardium, have also been demonstrated in many forms of adult heart disease and is a strong independent predictor of CVD risk in adults ${ }^{16}$. This was measured using a commercially available enzyme immunoassay test kit (BioCheck, Inc, Foster City, CA; Cat \# BC-1119). Intra- and inter- assay variations are less than 5 and $11 \%$, respectively.

Serum IL-6 and TNF: These cytokines were measured as research-based markers of systemic inflammation. Both IL-6 and TNFa have been correlated with adiposity in children 6-19 years of age. Association of these markers with SHS is less known, especially in young children. Plasma IL-6 levels were determined using a highly sensitive ELISA kit (R\&D systems, Minneapolis, MN; Cat \# DY206). Intra and inter- assay variations are less than 8 and $10 \%$, respectively.

\section{Biomarkers of oxidation:}

Protein malondialdehyde adducts: This is a measure of lipid peroxidation in serum ${ }^{17}$. Malondialdehyde is a highly reactive three-carbon dialdehyde byproduct of fatty acid peroxidation, which readily combines with several functional groups on molecules including proteins. We have found significant relationships between protein MDA and hair nicotine level in obese teenagers ${ }^{18}$. These were measured by OxiSelect ${ }^{\text {TM }}$ MDA Adduct competitive ELISA kits from Cell Biolabs, INC, San Diego, CA (Cat\#STA-832-5). The quantity of MDA adduct in samples was determined by comparing its absorbance with that of a predetermined MDA-BSA standard curve, and reported as pmol MDA $/ \mathrm{mL}$.

Total plasma anti-oxidant capacity: This measurement provides a non-specific measure of 'antioxidant reserve' in plasma and is thus an indication of the overall capability 
to counteract reactive oxidizing species and combat oxidative stress related diseases. It has been shown to be reduced in many settings of chronic inflammation/oxidation ${ }^{19}$. Both small molecule and protein antioxidants are measured in aggregate, and results are presented as $\mathrm{mM}$ Trolox equivalents (a reference reagent with quantitative antioxidant capacity). Commercial assay kits were from Biovision Research Products (Cat\#K274-100), Mountain View, $\mathrm{CA} . \mathrm{Cu}^{++}$was converted to $\mathrm{Cu}^{+}$by both small molecules and protein antioxidants in plasma samples. The levels of reduced $\mathrm{Cu}^{+}$ion were determined by the absorbance of a chelated colorimetric probe. Trolox was used as a standard antioxidant and total plasma antioxidant capacity was expressed as $\mathrm{mM}$ Trolox equivalents.

\section{Biomarkers of vascular endothelial stress:}

Soluble intercellular adhesion molecule-1 (s-ICAM1) was measured as a biomarker of endothelial stress and activation. Elevations in this marker have been documented in several settings of vascular injury (including coronary heart disease), and in relation to SHS exposure. ${ }^{13}$ Levels of s-ICAM-1 were determined using a sensitive commercially available ELISA kit (R\&D systems, Minneapolis, MN; Cat \# DY720). Intra and inter- assay variations are less than 5 and $10 \%$, respectively.

Circulating endothelial progenitor cells (EPCs): EPCs are bone marrow-derived stem cells that have the ability to differentiate into functional endothelial cells, circulate in the blood and home preferentially to sites of vascular or tissue injury, contributing significantly to both endothelial repopulation and neovascularization ${ }^{20}$. These cells have been recognized as a potential surrogate biological marker for vascular function and cumulative cardiovascular risk in adults ${ }^{21,22}$. Decreased EPC number and function have been shown in adult non-smokers exposed to $\mathrm{SHS}^{23}$ but have not been studied in relationship to SHS exposure in young children.

A volume of $50 \mu \mathrm{L}$ anticoagulated peripheral blood was incubated with $50 \mu \mathrm{L} 3 \%$ BSA in PBS (without $\mathrm{Ca}^{++}$and $\mathrm{Mg}^{++}$) at room temperature for $30 \mathrm{~min}$. In dark, fluorescence labeled antibodies (2.5 $\mu \mathrm{L}$ of each), PE-CD133, FITC-CD34, and PECy5-CD45, were added and incubated for $30 \mathrm{~min}$ at room temperature. FACS lysis buffer $(450 \mu \mathrm{L})$ was then added, and incubated for 30min at room temperature in dark. Samples were then analyzed on FACS Caliber flow cytometer, where total counts were $>400,000$ cells. Prevalence of CD34+/ CD45-, CD133+/CD45-, or CD34+/CD133+/CD45- cells was expressed as the percentage of total events.

\section{Statistical analyses:}

Statistical analyses were performed with Stata 13.1 (StataCorp. 2013 Stata Statistical Software: Release 13.0; Stata Corporation, College Station, TX). A $P$ value of 0.05 or less was considered statistically significant. Categorical variables were used to indicate sex (female $=1$, male=0); race (African American, White, Biracial, and Other Race); ethnicity (Hispanic or Latino=1, Non-Hispanic=0), insurance status (Medicaid benefits=1, All other insurance $=0$ ); maternal education (less than high school, high school graduate or GED, some college, or college graduate or higher). 
Continuous outcome variables were used for child's systolic blood pressure, diastolic blood pressure, systolic blood pressure \%, diastolic blood pressure \%, total cholesterol, LDL, HDL, fasting glucose, fasting insulin, CRP, IL-6, TNF- $a$, plasma protein malondialdehyde, total anti-oxidant capacity, soluble iCAM-1, and EPC prevalence. In multivariate regression analysis, fasting insulin, CRP levels, Il-6, TNF-a, plasma protein malondialdehyde and EPC prevalence were logarithmically transformed to improve the distribution of these variables. Age and BMI were also modeled as continuous measures. BMI was converted to a BMI zscore. A program available in Stata, zscore06 was used to calculate anthropometric z-scores. Length/height for-for-age, weight-for-height, BMI-for-age and weight-for-age Z-scores were calculated.

Bivariate associations between baseline demographic characteristics, SHS, and all cardiovascular outcomes were examined first using Pearson correlations. Multivariate linear regression with robust standard errors, clustered on family ID to account for the clustering of children within the same family was used to model each of the cardiovascular outcomes separately. All models included covariates for sex, race, ethnicity, insurance status, maternal education, age, and BMI z-score. Descriptive statistics are presented for all variables (means and SD for normally distributed and medians and interquartile ranges for skewed variables). Only significant models are presented for the multivariate models. Among the 149 children $2-5$ years of age that were included in the study, 10 had missing information on maternal education, with a final sample size $\mathrm{n}=139$ Additionally, eight children had missing information on systolic blood pressure, and systolic blood pressure \% and 9 had missing values for EPC prevalence. The models for CRP and TAC each had one additional missing case. Since models were run separately by outcome, the final sample size for blood pressure was $n=131$, TAC $n=138$, CRP $n=138$ and for $E P C n=122$. All other models presented had the full sample, minus those missing maternal education, $n=139$. Sensitivity analysis has confirmed that there are no significant differences with or without missing cases.

\section{RESULTS:}

\section{Population characteristics and sample distributions:}

Characteristics of the study subjects are shown in Table 1. The average age was $3.2 \mathrm{yrs}$, and the majority (82\%) was of lower socioeconomic status (insured through Medicaid), with a minority of mothers having a college degree or higher (14\%). One-third of the group was either overweight $(10.7 \%)$ or obese $(22.8 \%)$.

\section{Secondhand smoke exposure and hair nicotine levels:}

The enrolled participants were highly smoke exposed (Table 1); 66.4\% subjects lived with a smoker, and forty (26.8\%) lived with 2 or more smokers. Maternal smoking was present in sixty-two (42.3\%) of the enrolled subjects. Hair nicotine levels were not normally distributed, the median was $1.86 \mathrm{ng} / \mathrm{mg}$, with an average hair nicotine of $5.51 \mathrm{ng} / \mathrm{mg}$ (SD 8.43), and a wide range of exposure levels from $0.03 \mathrm{ng} / \mathrm{mg}$ (virtually no exposure) to 39.45 ng/mg. 
Table 2 presents the results of all outcome variables analyzed. Means and standard deviations are presented for the normally distributed variables, medians and interquartile ratios are presented for the variables with skewed distributions.

\section{Simple bivariate analyses: SHS vs. other measured cardiovascular risk variables.}

Preliminary bivariate analyses were performed using Pearson correlations, examining the associations between log-transformed hair nicotine vs. each other demographic and other measured variables ( $\mathrm{n}=131-139$ for each pairwise analysis). Log hair nicotine was significantly positively related to SBP (Pearson's $r=0.21$ ), SBP\% (Pearson's $r=0.30$ ), and DPB (Pearson's $r=0.17$ ), all significant at $<0.05$. Additionally, log hair nicotine was inversely related to total cholesterol (Pearson's $r=-0.23$ ) and HDL cholesterol (Pearson's $r$ $=-0.23$ ); both significant at the $<0.05$ level. Log hair nicotine was significantly positively related to $\log$ CRP (Pearson's $r=0.21$ ), and negatively related to EPC prevalence (Pearson's $r=-28$ ), both significant at the 0.05 level. There was no significant relationship between log hair nicotine and diastolic blood pressure percent, triglycerides, LDL and VLDL cholesterol, fasting glucose or insulin levels, IL6, TNFa, MDA, TAC, and s-ICAM1.

\section{Multivariate regression analyses:}

Separate multivariate linear regression analysis (clustered on family ID with robust standard errors) examined the relationship between log-transformed hair nicotine and all cardiovascular outcomes above. All models included controls for sex, age, race, ethnicity, method of payment, maternal education, and BMI z-score (Table 3). Log hair nicotine was independently significantly related to systolic blood pressure, systolic blood pressure percentile, HDL, CRP, TAC, and EPC prevalence. BMI z-score was also independently related to systolic blood pressure, systolic blood pressure percent, and HDL; however, no interaction effect was found between BMI and hair nicotine for any of the cardiovascular risk measures. Examining the clinical variables, each single log-increase in hair nicotine (corresponding to $2.72 \mathrm{ng} / \mathrm{mg}$ hair) was associated with an increase of $4.68 \mathrm{~mm} / \mathrm{Hg}$ for systolic blood pressure, a $0.92 \mathrm{mg} / \mathrm{L}$ increase in CRP, and a $4.42 \mathrm{mg} / \mathrm{dl}$ decrease in HDL. Regarding the research variables, each log-increase of hair nicotine was associated with a decrease of 0.68 units for EPC prevalence and 6.872 units (mM Trolox equivalents) in total anti-oxidant capacity.

\section{DISCUSSION:}

Although SHS is now recognized as a significant contributor to cardiovascular risks in older age groups, the toddler population has not been studied. The characteristics of the children we enrolled match a population of very high concern for SHS exposure (urban, African American, low-income $)^{24}$. More than half of the study participants lived with one or more smokers, and many of the participants had hair nicotine levels equivalent to published values associated with adult active smokers. This subgroup of US children is of very high concern regarding SHS exposure and later tobacco consumption and related disease risks.

In the present study, we evaluated an array of cardiovascular risk markers that encompassed clinical measures (blood pressure, lipids, glucose metabolism, inflammation) as well as three 
recognized aspects of tobacco-related mechanisms of injury in adults, 'research measures' (oxidation, vascular endothelial injury, and vascular repair). The apparently healthy toddler cohort had a wide range of values in these biomarkers. SHS exposure was independently linked to higher systolic blood pressure, HDL reduction, CRP elevation (a marker of inflammation), TAC reduction (a marker of oxidation) and EPC reduction (a marker of vascular repair). These relationships are all mechanistically relevant and apparently occurring simultaneously at a very young age. To our knowledge, this is one of the first studies to investigate the link between SHS exposure and cardiovascular disease risk in toddlers. These data extend prior work on the cardiovascular effects of tobacco exposure to a younger age group than previously studied.

Our findings demonstrate that health disparities noted during adulthood may at least be partially accounted for by early life SHS exposure. Epidemiological studies have shown the association between low socio-economic status during childhood and increased cardiovascular morbidity and mortality during adult life ${ }^{25}$. The lifetime health burden of youth who are SHS-exposed is bound to be significant. For example, a recent analysis of the Bogalusa Heart Study has shown that SHS exposure during childhood only showed a stronger association with cardiovascular risk than exposure in adulthood only ${ }^{26}$, suggesting an 'imprinting effect' of SHS-related disease risks. In addition to cumulative effects of years of exposure, children who grow up with smokers also are at greater risk of becoming active smokers due to the parental modeling of smoking behavior ${ }^{27}$. Obesity prevalence also varies inversely with $\mathrm{SES}^{28}$, and we noted an independent relationship between BMI z-score and systolic blood pressure and lower HDL among this group. Our goal should be to enhance our identification of the most-at-risk children and develop effective strategies to intervene and prevent adult cardiovascular disease.

One strength of this study is the focus on highly exposed, low-income, city-dwelling, children that may be the most vulnerable to physiological perturbations of SHS exposure. A convenience sample rather that a random or epidemiologically based sample is a potential limitation of this study, but we have no reason to believe that the relationships between SHS and outcomes investigated would be different due to sampling technique. We controlled for potential confounders including age, race, maternal education, method of payment, and BMI z-score, and acknowledge that this was a cross-sectional study, from which we can infer correlation but not causation. However, our findings are biologically plausible and align with previous data from older children, teens, and adults.

Another potential limitation is that we excluded children with persistent asthma. This exclusion would have biased our findings toward the null by excluding children with the most inflammation so that we may have underestimated the relationships between SHS and inflammation. Studies to understand the complete picture of cardiopulmonary risks and disease course patterns are needed, especially in young children.

In a very recent scientific statement, the American Heart Association has concluded that SHS is a compelling threat to cardiovascular health in children and that current evidence warrants ".... robust public health policy that embraces zero tolerance of childhood SHS exposure." Our study supports this view since we found an objectively measured marker of 
SHS (hair nicotine) was correlated to several variables already well-established as cardiovascular risk markers in adults. The fact that these relationships have been detected in toddlers, several decades before typical symptomatic heart disease, is alarming and may help to catalyze actions to enhance protection of young children from tobacco-related disease risks.

\title{
Supplementary Material
}

Refer to Web version on PubMed Central for supplementary material.

\section{Acknowledgments}

Funding Source: The research was supported by the American Academy of Pediatrics Julius B. Richmond Center of Excellence, (Co-PIs Judith A. Groner and John A. Bauer), which is funded by grants from the Flight Attendant Medical Research Institute and Legacy. The findings and conclusions are those of the authors and do not necessarily represent the official position of any of these institutions. No financial disclosures were reported by the authors of this paper.

Financial Disclosure Statement: We have no financial relationships to disclose.

\author{
Abbreviations: \\ SHS secondhand smoke \\ CRP C-reactive protein \\ EPC endothelial progenitor cell \\ S-ICAM1 soluble intercellular adhesion molecule
}

\section{REFERENCES:}

1. Homa DM, Neff LJ, King BA, Caraballo RS, Bunnell RE, Babb SD, et al. Vital signs: disparities in nonsmokers' exposure to secondhand smoke--United States, 1999-2012. MMWR Morb Mortal Wkly Rep. 2015;64(4):103-108. [PubMed: 25654612]

2. Jain RB. Trends in exposure to second hand smoke at home among children and nonsmoker adolescents. Sci Total Environ. 2016;542(Pt A):144-152. [PubMed: 26519575]

3. Quinto KB, Kit BK, Lukacs SL, Akinbami LJ. Environmental tobacco smoke exposure in children aged 3-19 years with and without asthma in the United States, 1999-2010. NCHS Data Brief. 2013(126):1-8.

4. Berenson GS, Pickoff AS. Preventive cardiology and its potential influence on the early natural history of adult heart diseases: the Bogalusa Heart Study and the Heart Smart Program. Am J Med Sci. 1995;310 Suppl 1:S133-138. [PubMed: 7503117]

5. He J, Vupputuri S, Allen K, Prerost MR, Hughes J, Whelton PK. Passive smoking and the risk of coronary heart disease--a meta-analysis of epidemiologic studies. N Engl J Med. 1999;340(12):920926. [PubMed: 10089185]

6. Benowitz NL. Cigarette smoking and cardiovascular disease: pathophysiology and implications for treatment. Prog Cardiovasc Dis. 2003;46(1):91-111. [PubMed: 12920702]

7. Glantz SA, Parmley WW. Passive smoking and heart disease. Mechanisms and risk. JAMA. 1995;273(13):1047-1053. [PubMed: 7897790]

8. Marano C, Schober SE, Brody DJ, Zhang C. Secondhand tobacco smoke exposure among children and adolescents: United States, 2003-2006. Pediatrics. 2009;124(5):1299-1305. [PubMed: 19841116] 
9. Groner JA, Huang H, Nicholson L, Kuck J, Boettner B, Bauer JA. Secondhand smoke exposure and hair nicotine in children: age-dependent differences. Nicotine Tob Res. 2012;14(9):1105-1109. [PubMed: 22193574]

10. Metsios GS, Flouris AD, Angioi M, Koutedakis Y. Passive smoking and the development of cardiovascular disease in children: a systematic review. Cardiol Res Pract. 2010;2011.

11. Wilkinson JD, Lee DJ, Arheart KL. Secondhand smoke exposure and C-reactive protein levels in youth. Nicotine Tob Res. 2007;9(2):305-307. [PubMed: 17365762]

12. Puranik R, Celermajer DS. Smoking and endothelial function. Prog Cardiovasc Dis. 2003;45(6): 443-458. [PubMed: 12800127]

13. Groner JA, Huang H, Nagaraja H, Kuck J, Bauer JA. Secondhand smoke exposure and endothelial stress in children and adolescents. Acad Pediatr. 2015;15(1):54-60. [PubMed: 25306212]

14. Neufeld EJ, Mietus-Snyder M, Beiser AS, Baker AL, Newburger JW. Passive cigarette smoking and reduced HDL cholesterol levels in children with high-risk lipid profiles. Circulation. 1997;96(5):1403-1407. [PubMed: 9315524]

15. Panagiotakos DB, Pitsavos C, Chrysohoou C, Skoumas J, Masoura C, Toutouzas P, et al. Effect of exposure to secondhand smoke on markers of inflammation: the ATTICA study. Am J Med. 2004;116(3):145-150. [PubMed: 14749157]

16. Joshi MS, Tong L, Cook AC, Schanbacher BL, Huang H, Han B, et al. Increased myocardial prevalence of C-reactive protein in human coronary heart disease: direct effects on microvessel density and endothelial cell survival. Cardiovasc Pathol. 2012;21(5):428-435. [PubMed: 22285194]

17. Marnett LJ. Lipid peroxidation-DNA damage by malondialdehyde. Mutat Res. 1999;424(1-2):8395. [PubMed: 10064852]

18. Groner JA, Huang H, Eastman N, Lewis L, Joshi MS, Schanbacher BL, et al. Oxidative Stress in Youth and Adolescents With Elevated Body Mass Index Exposed to Secondhand Smoke. Nicotine Tob Res. 2016;18(7):1622-1627. [PubMed: 26903500]

19. Fabian E, Poloskey P, Kosa L, Elmadfa I, Rethy LA. Nutritional supplements and plasma antioxidants in childhood asthma. Wien Klin Wochenschr. 2013;125(11-12):309-315. [PubMed: 23636616]

20. Yeh ET, Zhang S, Wu HD, Korbling M, Willerson JT, Estrov Z. Transdifferentiation of human peripheral blood CD34+-enriched cell population into cardiomyocytes, endothelial cells, and smooth muscle cells in vivo. Circulation. 2003;108(17):2070-2073. [PubMed: 14568894]

21. Hill JM, Zalos G, Halcox JP, Schenke WH, Waclawiw MA, Quyyumi AA, et al. Circulating endothelial progenitor cells, vascular function, and cardiovascular risk. N Engl J Med. 2003;348(7):593-600. [PubMed: 12584367]

22. Vasa M, Fichtlscherer S, Aicher A, Adler K, Urbich C, Martin H, et al. Number and migratory activity of circulating endothelial progenitor cells inversely correlate with risk factors for coronary artery disease. Circ Res. 2001;89(1):E1-7. [PubMed: 11440984]

23. Heiss C, Amabile N, Lee AC, Real WM, Schick SF, Lao D, et al. Brief secondhand smoke exposure depresses endothelial progenitor cells activity and endothelial function: sustained vascular injury and blunted nitric oxide production. J Am Coll Cardiol. 2008;51(18):1760-1771. [PubMed: 18452782]

24. Raghuveer G, White DA, Hayman LL, Woo JG, Villafane J, Celermajer D, et al. Cardiovascular Consequences of Childhood Secondhand Tobacco Smoke Exposure: Prevailing Evidence, Burden, and Racial and Socioeconomic Disparities: A Scientific Statement From the American Heart Association. Circulation. 2016.

25. Melchior M, Moffitt TE, Milne BJ, Poulton R, Caspi A. Why do children from socioeconomically disadvantaged families suffer from poor health when they reach adulthood? A life-course study. Am J Epidemiol. 2007;166(8):966-974. [PubMed: 17641151]

26. Chen W, Yun M, Fernandez C, Li S, Sun D, Lai CC, et al. Secondhand smoke exposure is associated with increased carotid artery intima-media thickness: the Bogalusa Heart Study. Atherosclerosis. 2015;240(2):374-379. [PubMed: 25875389] 
27. Selya AS, Dierker LC, Rose JS, Hedeker D, Mermelstein RJ. Risk factors for adolescent smoking: parental smoking and the mediating role of nicotine dependence. Drug Alcohol Depend. 2012;124(3):311-318. [PubMed: 22365898]

28. Clarke P, O’Malley PM, Johnston LD, Schulenberg JE. Social disparities in BMI trajectories across adulthood by gender, race/ethnicity and lifetime socio-economic position: 1986-2004. Int J Epidemiol. 2009;38(2):499-509. [PubMed: 18835869] 
Table 1:

Demographic data $(n=139)$

\begin{tabular}{|c|c|}
\hline Subject Characteristics & Mean \pm SD or $N(\%)$ \\
\hline Age, years & $3.22 \pm 1.06$ \\
\hline Female & $76(55 \%)$ \\
\hline \multicolumn{2}{|l|}{ Race } \\
\hline African-American & $76(54.68 \%)$ \\
\hline White & $32(23.02 \%)$ \\
\hline Multi-racial & $24(17.27 \%)$ \\
\hline Other & $7(5.03 \%)$ \\
\hline \multicolumn{2}{|l|}{ Ethnicity } \\
\hline Hispanic or Latino & $12(8.63 \%)$ \\
\hline \multicolumn{2}{|l|}{ Family Economic Status } \\
\hline Subject insured by Medicaid, n (\%) & $122(82 \%)$ \\
\hline \multicolumn{2}{|l|}{ Maternal Education, n (\%) } \\
\hline Less than high school & $44(31.65 \%)$ \\
\hline High school or GED & $35(25.18 \%)$ \\
\hline Some college & $41(29.50 \%)$ \\
\hline \multirow[t]{2}{*}{ College graduate } & $19(13.67 \%)$ \\
\hline & Mean $\pm S D$ or median (range) \\
\hline SHS exposure: hair nicotine (ng/mg) & $5.51 \pm 8.43$ \\
\hline Hair nicotine median, range (ng/mg) & $1.86(0.025-39.45)$ \\
\hline $\mathrm{BMI}, \mathrm{kg} / \mathrm{m}^{2}$ & $16.98 \pm 2.28$ \\
\hline BMI, z-score & $0.90 \pm 1.39$ \\
\hline
\end{tabular}


Table 2.

\begin{tabular}{|c|c|c|c|}
\hline \multicolumn{4}{|c|}{$\rightarrow \quad$ Measured variables } \\
\hline \multirow{6}{*}{ 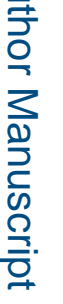 } & & Mean \pm SD & Sample N \\
\hline & \multicolumn{3}{|l|}{ Normally distributed variables } \\
\hline & Systolic Blood Pressure, $\mathrm{mm} \mathrm{Hg}$ & $95.59 \pm 10.53$ & 131 \\
\hline & Diastolic Blood Pressure, $\mathrm{mm} \mathrm{Hg}$ & $59.70 \pm 8.21$ & 131 \\
\hline & Systolic Blood Pressure, $\%$ & $59.77 \pm 27.98$ & 131 \\
\hline & Diastolic Blood Pressure, $\%$ & $74.83 \pm 20.00$ & 131 \\
\hline \multirow{14}{*}{ 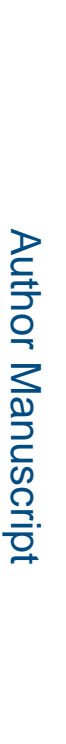 } & Total Cholesterol, mg/dL & $146.46 \pm 26.82$ & 139 \\
\hline & Low Density Lipoprotein, mg/dL & $84.35 \pm 23.58$ & 139 \\
\hline & High Density Lipoprotein, mg/dL, & $48.73 \pm 13.28$ & 139 \\
\hline & Fasting blood glucose, $\mathrm{mg} / \mathrm{dl}$ & $80.68 \pm 7.28$ & 139 \\
\hline & Total anti-oxidant capacity, mm Trolox equivalent & $102.59 \pm 22.65$ & 138 \\
\hline & Soluble ICAM-1, ng/mL & $206.89 \pm 64.96$ & 138 \\
\hline & & Median (IQR) & \\
\hline & Skewed distribution variables & & \\
\hline & Fasting insulin, uIU/mL & $6.46(5.33-8.33)$ & 138 \\
\hline & C-Reactive Protein, mg/L & $0.22(0.04-1.27)$ & 138 \\
\hline & Interleukin-6, ng/L & $13.31(4.69-113.70)$ & 138 \\
\hline & TNF-a, ng/L & $148.66(76.54-1026.86)$ & 138 \\
\hline & Plasma protein malondialdehyde, pmol MDA/mL & $18.8(15.1-22.8)$ & 138 \\
\hline & Circulating CD34+ Cells, \% of total events & $0.0086(0.0042-0.0156)$ & 122 \\
\hline
\end{tabular}


3.

Multivariate Linear Regression Analysis of Associations with Logarithmically Transformed Hair Nicotine Levels

\begin{tabular}{|l|l|l|l|l|}
\hline & & \multicolumn{3}{|c|}{ Log Hair Nicotine } \\
\hline Outcome & Model N & b & SE b & \multicolumn{1}{|l|}{} \\
\hline Systolic blood pressure & 139 & 4.68 & 1.84 & .013 \\
\hline Systolic blood pressure \% & 139 & 15.32 & 5.21 & .004 \\
\hline CRP & 138 & 0.92 & 0.35 & .012 \\
\hline HDL & 139 & -4.42 & 1.82 & .017 \\
\hline Total anti-oxidant capacity & 138 & -6.72 & 3.08 & .032 \\
\hline EPC prevalence & 122 & -0.39 & 0.17 & .028 \\
\hline
\end{tabular}

Notes: Each row represents a separate regression model. Each regression model is adjusted for sex, age, race, ethnicity, insurance payment, maternal education, and BMI z-score is clustered on family ID and robust standard errors. 